\title{
Programa Academia da Saúde: um olhar quantitativo das adesões entre 2011 a 2017
}

\section{The Health Academy Program in Brazil: a quantitative view of adhesions between 2011 to 2017}

\section{AUTORES \\ Dalila Tusset $^{1}$ (D) \\ Mabel Olkoski (i) \\ Edgar Merchan-Hamann ${ }^{1}$ (iD) \\ Paulo Carlos Du Pin Calmon 3 (D) \\ Leandro dos Santos ${ }^{4}$ (D) \\ 1 Universidade de Brasilia, Faculdade de Ciências da Saúde, Brasília, Distrito Federal, Brasil. \\ 2 Universidade do Estado de Santa Catarina, Departamento de Produção Animal, Lages, Santa Catarina, Brasil. \\ 3 Universidade de Brasilia, Brasilia, Distrito Federal, Brasil. \\ 4 Universidade Estadual de Londrina, Grupo de Estudo e Pesquisa em Metabolismo Nutrição e Exercício, Londrina, Paraná, Brasil.}

\section{CONTATO}

\section{Dalila Tusset}

dalilatusset@gmail.com

Rua Joaquim Esperidião, 730, Anápolis,

Goiás, Brasil.

CEP: 75113-420.

DOI

$10.12820 /$ rbafs. $25 \mathrm{e} 0165$

\section{(cc) BY}

Este trabalho está licenciado com uma Licença Creative Commons - Atribuição 4.0 Internacional.

\begin{abstract}
RESUMO
O objetivo foi analisar a distribuição das adesões municipais ao Programa Academia da Saúde (PAS) no território nacional e o impacto da alteração do tipo de financiamento das obras no quantitativo de adesões. Foram utilizados dados secundários disponibilizados pelo Ministério da Saúde (MS) referentes às adesões ocorridas de 2011 a 2017 para compor as variáveis do estudo. Foram realizadas análises descritiva e inferencial, empregando-se o teste t pareado de Student para verificar diferenças entre adesões em diferentes anos e modalidades de financiamento (emenda parlamentar-EP e MS). Os resultados mostram que a maioria das macrorregiões apresentou mais de $50 \%$ de municípios participantes (municípios-PAS). As regiões Nordeste e Sudeste tiveram mais adesões, mas a região Norte destacou-se com o maior quantitativo de municípios-PAS proporcionalmente ao total de municípios. O período de maior expansão foi de 2011 a 2013. O número de adesões e municípios-PAS nos cinco anos de financiamento exclusivo por EP não superou o quantitativo aprovado de $2011 \mathrm{a}$ 2012. Nos primeiros anos de financiamento por EP, ocorreu concentração das adesões nos mesmos municípios. Todas as macrorregiões apresentaram mais de $60 \%$ de obras concluídas, entretanto foi baixo o percentual de academias com custeio federal dentre as adesões aptas ao recebimento. Concluímos que o PAS está bem distribuído em todo o país, com diferentes situações de implantação. $\mathrm{O}$ período de maior expansão do programa foi em 2011 e 2012, quando as obras eram financiadas com recurso do MS. De 2013 a 2017, com financiamento exclusivo por EP, o quantitativo de adesões não superou o observado nos dois primeiros anos.
\end{abstract}

Palavras-chave: Saúde pública; Promoção da saúde; Financiamento governamental; Doença crônica. ABSTRACT

This study aimed to analyze the adhesions of the Health Academy Program (HAP) in the country and the impact of the funding type alteration for the facilities building. Secondary data provided by the Ministry of Health regarding the adhesions from 2011 to 2017 were used to compose the variables. Descriptive and inferential analyses were conducted, using the Student's paired t-test to verify differences between the number of adhesions to the HAP in different years and funding types. Most regions had more than $50 \%$ of participating municipalities. Northeast and Southeast presented greater adhesions numbers concerning the other regions, but the North was highlighted with the largest quantity proportional to the number of municipalities. The greatest expansion was from 2011 to 2013. The number of facilities and municipalities enrolled in the HAP during the years of exclusive funding by parliamentary amendments did not exceed the amount approved from 2011 to 2012. There was a concentration of adhesions in the same municipalities in 2012 and 2013 when the parliamentary amendments were included. In all regions, the rate of facility building completion was above 60\%, however the percentage of facilities with the maintenance funding among those able to receive it is low. In conclusion, the HAP has been largely implemented in the country, with a variety of implementation status. From 2011 to 2012 occurred the greatest expansion of the HAP, period that the facilities where financed with funds from the Ministry of Health. In the others hand, from 2013 to 2017, with exclusive funding by EP, the number of facilities did not exceed that observed in the first two years.

Keywords: Public health; Health promotion; Government financing; Chronic disease.

\section{Introdução}

Um dos problemas globais de grande relevância no cenário epidemiológico é constituído pela carga de doença atribuída às doenças crônicas não transmissíveis $(\mathrm{DCNT})^{1}$. Esse conjunto de morbidades são evitáveis mediante a modificação de fatores de risco, como sedentarismo, tabagismo, alcoolismo e alimentação inadequada ${ }^{2}$. Ao enfrentar esse quadro, o modelo biomédico mostrou-se insuficiente, oportunizando a discussão que prioriza a promoção da saúde (PS) como uma estratégia de ação política em saúde pública de melhor custo-efetividade ${ }^{3}$. 
A PS recebeu diversos olhares e possibilidades de concretude nos sistemas de saúde ${ }^{4}$. No Brasil, foi institucionalizada como política em 2006, levando à formulação de diversos programas. $O$ processo de construção da política nacional de PS (PNPS) contou com duas versões oficiais, delineadas em meio a disputas ideológicas e políticas ${ }^{5,6}$. Mesmo com restrições aos enfoques em mudanças de estilos de vida e responsabilização individual, a maioria das ações na primeira versão da PNPS seguiram essa orientação ${ }^{6}$. De 2013 a 2014, a PNPS foi amplamente revisada com a participação do governo e de diferentes segmentos sociais ${ }^{5}$. O viés comportamentalista parece ter sido superado por um objetivo mais amplo, envolvendo o ideário da PS pautado em equidade e redução de riscos e vulnerabilidades à saúde atrelados aos seus determinantes sociais ${ }^{7}$.

A atividade física, incluída nas duas versões da PNPS, foi, desse modo, inserida no Sistema Único de Saúde $(\mathrm{SUS})^{8}$. Influíram nessa inserção diversas iniciativas, como a Estratégia Global para Alimentação, Atividade Física e Saúde 6 , o Plano de Ações Estratégicas para o Enfrentamento das Doenças Crônicas Não Transmissíveis (DCNT) 2011 a $2022^{9}$ e também resultados de ações integradas à atenção primária em nível local ${ }^{6}$.

Um dos principais programas voltados para a abordagem da PS é o Programa Academia da Saúde (PAS), instituído em 2011 no âmbito da atenção primária ${ }^{10}$ como um potencial meio para incorporação de ações de PS no enfrentamento de DCNT e, principalmente, da inclusão da atividade física no SUS. O PAS contemplou a implantação de infraestrutura específica e quadro de pessoal qualificado e, em 2013, teve seu arcabouço normativo e operacional redefinido para que atores políticos e técnicos compreendessem sua inserção na rede de atenção, considerando os modos de vida das pessoas e não apenas mudanças comportamentais como foco da ação governamental ${ }^{10}$.

Para o seu desenvolvimento, o PAS contou inicialmente com financiamento exclusivo do MS, porém não foi considerado no orçamento impositivo. Em 2012, o legislativo reconheceu que o PAS não poderia mais ser financiado pelo orçamento MS por ser ação de PS, e, portanto, de responsabilidade intersetorial ${ }^{11}$. Essa interpretação foi acatada pelo executivo, com a instituição do financiamento da infraestrutura por emendas parlamentares individuais (EP). Todavia, o MS manteve o financiamento da manutenção, ou seja, o custeio mensal. A partir desse episódio, concedeu-se ao legislativo o poder político na alocação das academias da saú- de no território nacional por meio da disponibilização de recursos às prefeituras municipais.

Recentemente, verificamos que as adesões ao PAS têm sido concedidas por meio de emenda parlamentar e os municípios com os piores indicadores socioeconômicos e de DCNT são os que menos se beneficiam com as adesões, no entanto, pouco se sabe sobre a distribuição territorial e os números de adesões ao $\mathrm{PAS}^{12}$. $\mathrm{Co}^{-}$ nhecer essas dimensões do programa é relevante tanto para atores governamentais como para os interessados em novas propostas ou em investigações científicas. $\mathrm{O}$ PAS é uma entre muitas iniciativas públicas destinadas ao bem-estar social que envolvem obras públicas, questão complexa na realidade brasileira. Dessa forma, o objetivo do presente estudo foi analisar a distribuição das adesões municipais ao PAS no território nacional e o impacto da alteração do tipo de financiamento das obras no quantitativo de adesões.

\section{Métodos}

O estudo caracteriza-se como uma pesquisa comparativa, transversal com a utilização de dados secundários obtidos junto ao MS, provenientes do Sistema de Monitoramento de Obras, dos bancos de controle administrativo da gestão federal do PAS e do Sistema de Apoio à Implementação de Políticas em Saúde. As unidades de análise são os municípios brasileiros e suas adesões ao PAS ocorridas entre 2011 a 2017. Foram consideradas adesões, as academias da saúde solicitadas pela gestão municipal e aprovadas pelo governo federal, independentemente da fase da implantação.

O governo federal viabiliza a implementação do PAS por meio dos seguintes componentes ${ }^{13}$ : a) transferência de recursos financeiros à gestão municipal para a construção das academias da saúde; b) transferência mensal de custeio para financiar o funcionamento do PAS. O custeio é destinado para as academias construídas e para academias que surgiram mediante o processo de adesão por "similaridade", ou seja, quando o município recebe aprovação do pedido de implementação do PAS por possuir os recursos de pessoal e físicos necessários ${ }^{13}$.

A alteração do financiamento das obras do PAS fez com que determinados municípios obtivessem adesões com financiamento do MS (AMS) e outras adesões por EP (AEP). No Quadro 1, apresenta-se a forma do financiamento das obras de acordo com o ano no período estudado.

Os dados obtidos para a condução deste estudo são relativos às adesões "por similaridade" e "por obra", 
Quadro 1 - Financiamento das obras do Programa Academia da Saúde de 2011 a 2017.

\begin{tabular}{|llllllll|}
\hline \multicolumn{1}{|c}{ Ano } & 2011 & 2012 & 2013 & 2014 & 2015 & 2016 & 2017 \\
\hline Financiamento & Sim & Sim & Não & Não & Não & Não & Não \\
Emento do Ministério da Saúde & Não & Sim & Sim & Sim & Sim & Sim & Sim \\
\hline
\end{tabular}

abrangendo a aprovação de custeio para cada adesão e o tipo de financiamento em caso de obra (MS e EP), tendo como referência abril de 2018. A partir desses dados, foram compostas as seguintes variáveis descritivas: adesões aprovadas, obras concluídas, adesões custeadas, adesões canceladas, municípios participantes (municípios-PAS) e municípios desistentes.

Além disso, foram construídas as variáveis razão entre o número de municípios-PAS e o número de adesões por macrorregião, a qual permite identificar se houve concentração de adesões nos mesmos municípios ao longo dos anos (representado por quociente mais baixos), e a variável razão entre o número de adesões e número de municípios de cada macrorregião, utilizada para verificar a proporcionalidade das adesões nas macrorregiões.

O software $R$ foi utilizado para a preparação do banco de dados e a condução das análises descritiva e inferencial. Utilizou-se o teste t de Student para dados pareados para a análise comparativa do total acumulado de adesões por ano e períodos. Para definição dos grupos de municípios a serem comparados, adotou-se como critério o tipo de financiamento anual, a saber:

- Municípios ( $n=135)$ que receberam adesão tanto em 2011 (apenas AMS) quanto em 2012 (AMS e AEP): comparação entre as médias do total em 2011 e em 2012 (2011-2012);

- Municípios $(\mathrm{n}=75)$ que receberam adesão tanto em 2012 quanto em 2013 (apenas AEP): comparação entre as médias do total em 2012 e em 2013 (2012-2013);

- Municípios ( $\mathrm{n}=232$ ) que receberam adesão em ambos dos seguintes períodos: comparação entre as médias do total acumulado em 2011-2012 (AMS e AEP) e em 2013-2017 (apenas AEP);

- Municípios ( $\mathrm{n}=88$ ) que receberam adesão em ambos dos seguintes períodos: comparação entre as médias do total acumulado em 2011-2013 (AMS e AEP) e em 2014-2017 (apenas AEP).

Dispensou-se a apreciação por Comitê de Ética em Pesquisa, pois os dados utilizados são secundários de domínio público.

\section{Resultados}

Os municípios contemplados com o PAS de 2011 a 2017, por tipo de adesão, constam na Figura 1, demonstrando a predominância de municípios com adesões por obras. Mais da metade dos municípios brasileiros $(\mathrm{n}=2.864)$ foram contemplados com o PAS, totalizando 4.087 adesões. A maioria das macrorregiões possuía acima de $50 \%$ de municípios-PAS, com exceção do Sudeste (44\%). A região Norte apresentou o maior percentual (58\%), seguido das regiões Nordeste (56\%), Centro-Oeste (55\%) e Sul (51\%). Também pertencem à região Norte os estados com a menor e a maior proporção de municípios-PAS (Amazonas, 21\% e Acre, 100\%). Roraima e Mato Grosso do Sul também se destacaram com $80 \%$ de municípios-PAS (Tabela 1).

Em relação ao total de adesões (4.087), as obras correspondem a $97 \%$ (3.966) e as similares apenas 3\% ( $\mathrm{n}=$ 121) - Tabela 1. Nordeste e Sudeste alocam os maiores quantitativos das adesões em nível nacional. As duas macrorregiões contemplam $91 \%$ das similares (Sudeste $\mathrm{n}=69 ;$ Nordeste $\mathrm{n}=51$ ) e $62 \%$ das obras (Sudeste $\mathrm{n}$ = 1127; Nordeste $n=1338)-$ Tabela 1 . Considerando a proporcionalidade entre o número de adesões e de municípios das macrorregiões, conforme apresentado na Tabela 1 , a região Norte apresentou a maior razão $(0,85)$ e a região Sul a menor $(0,65)$.

O cenário de implantação altera-se quando se consideram as proporções de adesões canceladas, 9\% das adesões (389 de 4.087) e 8\% (235 de 2.864) dos municípios-PAS (Tabela 1). Sudeste e Nordeste tiverem, respectivamente, os maiores e os menores percentuais de adesões canceladas (19\% versus 3\%) e municípios desistentes (17\% versus $3 \%)$. Espírito Santo foi o estado com maior percentual (35\%) de municípios que deixaram de participar do PAS em função dos cancelamentos. Apenas três estados (Acre, Roraima e Alagoas) não tiveram cancelamentos de adesões, e o Distrito Federal destacou-se com 94\% das adesões canceladas (Tabela 1).

Quanto aos resultados da situação das obras ativas, cerca de $70 \%$ das obras nacionais estavam concluídas e todas as macrorregiões apresentaram percentuais de fi- 


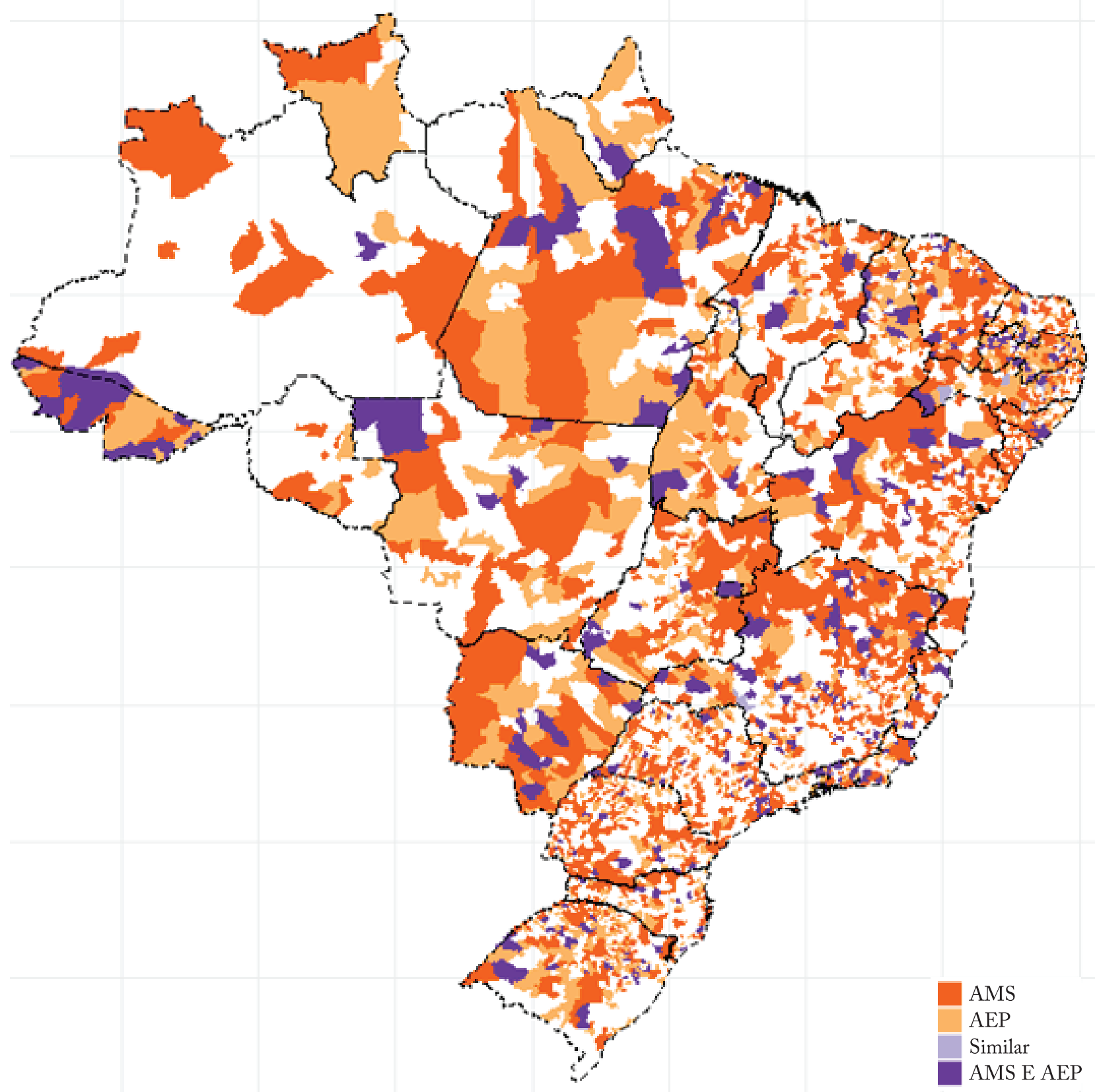

Figura 1 - Municípios que participaram da adesão ao Programa Academia da Saúde, por tipo de adesão, de 2011 a 2017. Legenda: $\mathrm{AMS}$ = adesão com financiamento do Ministério da Saúde; AEP = adesão por emenda parlamentar.

nalização acima de 60\%, com maior percentual do Sul (81\%) e menor do Nordeste (63\%) - Tabela 1. Em relação aos estados, os maiores percentuais de conclusão das academias foram em Rondônia (92\%), Acre (87\%), Paraná e Santa Catarina (85\%), já Rio de Janeiro (40\%) e Amapá (42\%) apresentaram os menores percentuais (Tabela 1$)$.

Cerca de $30 \%$ das adesões estavam com custeio aprovado, incluindo similares $(\mathrm{n}=121)$ e obras finalizadas $(n=684)$ - Tabela 1 . Considerando o total de obras finalizadas ( $n=2.478$ ), ou seja, que já possuem a estrutura disponível para o funcionamento do PAS, o percentual de custeio foi baixo, $28 \%(\mathrm{n}=684)$. Entre as macrorregiões não houve variação importante quanto ao custeio, mínimo de $24 \%$ no Norte e máximo de $30 \%$ no Nordeste (Tabela 1).

Conforme exposto no quadro 2, predominaram as AMS $(53 \%, n=2.178)$. O financiamento do MS também responsável pela maioria das adesões no Nordeste (52\%, 693 de 1.338), Centro-Oeste (53\%, 182 de 343), Sudeste $(63 \%, 711$ de 1127) e Sul (55\%, 432 de 775). 
Tabela 1 - Distribuição das adesões do Programa Academia da Saúde (PAS) aprovadas de 2011 a 2017, por unidade da federação e situação da adesão.

\begin{tabular}{|c|c|c|c|c|c|c|c|c|c|}
\hline \multirow{2}{*}{ UF } & & \multicolumn{3}{|c|}{ Município-PAS } & \multicolumn{5}{|c|}{ Adesão } \\
\hline & & $\frac{\text { Participante }}{\mathrm{n}(\%)}$ & $\frac{\text { Desistente }}{\mathrm{n}(\%)}$ & $\begin{array}{c}\text { Aprovada } \\
\text { (similar e obra) }\end{array}$ & $\frac{\text { Similar }}{\mathrm{n}}$ & $\frac{\text { Obra }}{n}$ & $\begin{array}{c}\text { Obra } \\
\text { Cancelada* }\end{array}$ & $\begin{array}{c}\begin{array}{c}\text { Obra } \\
\text { concluída** }^{* *}\end{array} \\
\mathrm{n}(\%)\end{array}$ & $\begin{array}{c}\text { Obra com } \\
\text { Custeio }^{* * * * *} \\
\mathrm{n}(\%)\end{array}$ \\
\hline \multirow{6}{*}{ NO } & $\mathrm{AC}$ & $22(100)$ & 0 & 45 & 0 & 45 & 0 & $39(87)$ & $5(11)$ \\
\hline & $\mathrm{AM}$ & $13(21)$ & $2(15)$ & 21 & 0 & 21 & $9(43)$ & $8(67)$ & $2(17)$ \\
\hline & $\mathrm{AP}$ & $11(69)$ & $1(9)$ & 22 & 0 & 22 & $3(14)$ & $8(42)$ & 0 \\
\hline & $\mathrm{PA}$ & $96(67)$ & $7(7)$ & 152 & 0 & 152 & $8(5)$ & $94(65)$ & $27(29)$ \\
\hline & RO & $12(23)$ & $1(8)$ & 13 & 0 & 13 & $1(8)$ & $11(92)$ & $1(1)$ \\
\hline & $\mathrm{RR}$ & $12(80)$ & 0 & 17 & 0 & 17 & 0 & $11(65)$ & $1(1)$ \\
\hline \multirow{12}{*}{$\mathrm{NE}$} & TO & $96(69)$ & $4(4)$ & 114 & 1 & 113 & $6(5)$ & $74(69)$ & $22(30)$ \\
\hline & Subtotal & $262(58)$ & $15(5)$ & 384 & 1 & 383 & $27(7)$ & $245(69)$ & $58(24)$ \\
\hline & $\mathrm{AL}$ & $61(60)$ & 0 & 92 & 0 & 92 & 0 & $45(49)$ & $19(42)$ \\
\hline & $\mathrm{BA}$ & $197(47)$ & $6(3)$ & 244 & 1 & 243 & $7(3)$ & $174(74)$ & $61(35)$ \\
\hline & $\mathrm{CE}$ & $113(61)$ & $3(2)$ & 149 & 0 & 149 & $6(4)$ & $115(80)$ & $25(22)$ \\
\hline & MA & $93(43)$ & $4(4)$ & 132 & 0 & 132 & $4(3)$ & $74(58)$ & $17(23)$ \\
\hline & $\mathrm{PB}$ & $176(79)$ & $5(2)$ & 238 & 0 & 238 & $5(2)$ & $144(62)$ & $52(36)$ \\
\hline & $\mathrm{PE}$ & $116(63)$ & $5(4)$ & 209 & 48 & 161 & $7(4)$ & $79(51)$ & $29(37)$ \\
\hline & PI & $108(48)$ & $3(2)$ & 156 & 0 & 156 & $3(2)$ & $114(75)$ & $27(24)$ \\
\hline & $\mathrm{RN}$ & $90(54)$ & $4(4)$ & 118 & 1 & 117 & $4(3)$ & $70(62)$ & $18(26)$ \\
\hline & SE & $44(59)$ & $5(11)$ & 51 & 1 & 50 & $6(12)$ & $28(64)$ & $8(28)$ \\
\hline & Subtotal & $998(56)$ & $35(3)$ & 1389 & 51 & 1338 & $42(3)$ & 843 (63) & $256(30)$ \\
\hline \multirow{5}{*}{$\mathrm{CO}$} & DF & 1 & 1 & 17 & 0 & 17 & $16(94)$ & 0 & 0 \\
\hline & GO & $127(52)$ & $3(2)$ & 154 & 0 & 154 & $3(2)$ & $107(71)$ & $41(38)$ \\
\hline & MS & $63(80)$ & $6(9)$ & 95 & 0 & 95 & $6(6)$ & $64(72)$ & $9(14)$ \\
\hline & MT & $64(45)$ & $3(5)$ & 77 & 0 & 77 & $4(5)$ & $53(73)$ & $12(23)$ \\
\hline & Subtotal & $255(55)$ & $13(5)$ & 343 & 0 & 343 & $29(8)$ & $224(71)$ & $62(28)$ \\
\hline \multirow{5}{*}{ SE } & ES & $31(40)$ & $11(35)$ & 50 & 6 & 44 & $18(41)$ & $15(58)$ & $1(6)$ \\
\hline & MG & $376(44)$ & 41 (11) & 558 & 50 & 508 & $62(12)$ & 304 (68) & $112(37)$ \\
\hline & RJ & $63(68)$ & $17(27)$ & 190 & 0 & 190 & $40(21)$ & $60(40)$ & $6(10)$ \\
\hline & SP & $273(42)$ & $55(20)$ & 398 & 13 & 385 & 104 (27) & $214(76)$ & $62(29)$ \\
\hline & Subtotal & $743(44)$ & 124 (17) & 1196 & 69 & 1127 & 224 (19) & $593(66)$ & $168(28)$ \\
\hline \multirow{4}{*}{ SU } & PR & 197 (49) & $14(7)$ & 228 & 0 & 228 & $17(7)$ & $179(85)$ & $51(28)$ \\
\hline & RS & $287(58)$ & $26(9)$ & 400 & 0 & 400 & $40(10)$ & $278(77)$ & $50(18)$ \\
\hline & $\mathrm{SC}$ & $122(41)$ & $8(6)$ & 147 & 0 & 147 & $10(7)$ & $116(85)$ & $39(34)$ \\
\hline & Subtotal & $606(51)$ & $48(8)$ & 775 & 0 & 775 & $67(10)$ & $573(81)$ & $140(24)$ \\
\hline Brasil & Total & $2864(51)$ & $235(8)$ & 4087 & 121 & 3966 & $389(9)$ & $2478(69)$ & $684(28)$ \\
\hline
\end{tabular}

*Percentual calculado com base no número de obras; **Percentual calculado com base no número de obras ativas, ou seja, não canceladas; ****Percentual calculado com base no número de obras concluídas.

As AEP foram a maioria apenas no Norte (59\%, 223 de 383). Houve um crescimento notável em termos de adesões (104\%) e de municípios-PAS (73\%) de 2011 a 2013. Entre as macrorregiões, em 2017, Sul concentrava menos $(0,8)$ e o Sudeste mais $(0,6)$ adesões nos mesmos municípios. Em nível nacional, essa concentração foi verificada no período 2011-2013 (de 0,9 para 0,7), ou seja, municípios que já eram participantes receberam mais academias em anos seguintes (Quadro 2).

Comparativamente, 2012 e 2013 apresentaram valores médios de adesão superiores aos anos anterio- res, sendo de 3,15 e 3,25 adesões, respectivamente (p $<0.001)$ - Figura 2. Essa diferença também foi encontrada quando comparadas as médias nos períodos 2011/2013 a 2014/2017 (p < 0,041) - Figura 3.

\section{Discussão}

Os principais resultados apresentados demonstram que metade dos municípios brasileiros tiveram a implantação do PAS, com expressiva participação das AMS, a maioria das macrorregiões apresentaram acima de 50\% de municípios-PAS e o menor volume de adesões ocor- 
Quadro 2 - Distribuição periódica e anual das adesões e dos municípios participantes do Programa Academia da Saúde por tipo de adesão e macrorregião, de 2011 a 2017.

\begin{tabular}{|c|c|c|c|c|c|c|c|c|c|c|c|c|c|c|c|}
\hline \multirow[b]{3}{*}{$\mathrm{UF}$} & \multirow[b]{3}{*}{ M } & \multicolumn{5}{|c|}{ Adesões por períodos (n) } & \multicolumn{9}{|c|}{ Adesões anuais cumulativas (n) } \\
\hline & & \multicolumn{3}{|c|}{$2011-2012$} & \multicolumn{2}{|c|}{$2013-2017^{*}$} & \multicolumn{3}{|c|}{2011} & \multicolumn{3}{|c|}{2012} & \multicolumn{3}{|c|}{2013} \\
\hline & & AS & AMS & $\mathrm{AEP}$ & AS & AEP & $\mathrm{T}$ & $\mathrm{MP}$ & $\bar{R}$ & $\bar{T}$ & MP & $\bar{R}$ & $\overline{\mathrm{T}}$ & $\mathrm{MP}$ & $\mathrm{R}$ \\
\hline $\mathrm{NE}$ & 1794 & 20 & 693 & 215 & 31 & 430 & 592 & 531 & 0,9 & 928 & 756 & 0,8 & 1242 & 924 & 0,7 \\
\hline $\mathrm{NO}$ & 450 & 0 & 160 & 90 & 1 & 133 & 119 & 106 & 0,9 & 250 & 191 & 0,8 & 362 & 254 & 0,7 \\
\hline $\mathrm{CO}$ & 467 & 0 & 182 & 72 & 0 & 89 & 149 & 134 & 0,9 & 254 & 195 & 0,8 & 333 & 251 & 0,8 \\
\hline $\mathrm{SE}$ & 1668 & 25 & 711 & 150 & 44 & 266 & 598 & 453 & 0,8 & 886 & 628 & 0,7 & 1111 & 725 & 0,7 \\
\hline $\mathrm{SU}$ & 1191 & 0 & 432 & 165 & 0 & 178 & 386 & 353 & 0,9 & 597 & 493 & 0,8 & 726 & 583 & 0,8 \\
\hline $\mathrm{BR}$ & 5570 & 45 & 2178 & 692 & 76 & 1096 & 1844 & 1577 & 0,9 & 2915 & 2263 & 0,8 & 3774 & 2737 & 0,7 \\
\hline \multicolumn{16}{|c|}{ Adesões anuais cumulativas (n) } \\
\hline & & \multicolumn{3}{|c|}{2014} & \multicolumn{3}{|c|}{2015} & \multicolumn{3}{|c|}{2016} & \multicolumn{3}{|c|}{2017} & & \\
\hline UF & $\mathrm{M}$ & $\overline{\mathrm{T}}$ & $\mathrm{MP}$ & $\mathrm{R}$ & $\overline{\mathrm{T}}$ & MP & $\mathrm{R}$ & $\mathrm{T}$ & $\mathrm{MP}$ & $\mathrm{R}$ & $\mathrm{T}$ & $\mathrm{MP}$ & $\mathrm{R}$ & & \\
\hline $\mathrm{NE}$ & 1794 & 1274 & 941 & 0,7 & 1299 & 956 & 0,7 & 1337 & 977 & 0,7 & 1389 & 998 & 0,7 & & \\
\hline $\mathrm{NO}$ & 450 & 362 & 254 & 0,7 & 364 & 255 & 0,7 & 376 & 257 & 0,7 & 384 & 262 & 0,7 & & \\
\hline $\mathrm{CO}$ & 467 & 336 & 252 & 0,8 & 336 & 252 & 0,8 & 338 & 253 & 0,7 & 343 & 255 & 0,7 & & \\
\hline $\mathrm{SE}$ & 1668 & 1159 & 733 & 0,6 & 1170 & 735 & 0,6 & 1184 & 739 & 0,6 & 1196 & 743 & 0,6 & & \\
\hline $\mathrm{SU}$ & 1191 & 749 & 597 & 0,8 & 758 & 600 & 0,8 & 764 & 603 & 0,8 & 775 & 606 & 0,8 & & \\
\hline BR & 5570 & 3880 & 2777 & 0,7 & 3927 & 2798 & 0,7 & 3999 & 2829 & 0,7 & 4087 & 2864 & 0,7 & & \\
\hline
\end{tabular}

$\mathrm{M}$ = municípios na macrorregião; $\mathrm{AS}$ = número de adesão por similaridade; $\mathrm{AMS}$ = número de adesão por programa; $\mathrm{AEP}=$ número de adesão por emenda; $\mathrm{T}$ = número total de adesões; $\mathrm{MP}$ = número de municípios participantes; $\mathrm{R}$ = razão entre municípios participantes e adesões $(\mathrm{MP} / \mathrm{T}) .{ }^{*}$ Nesse período não houve adesão por programa.

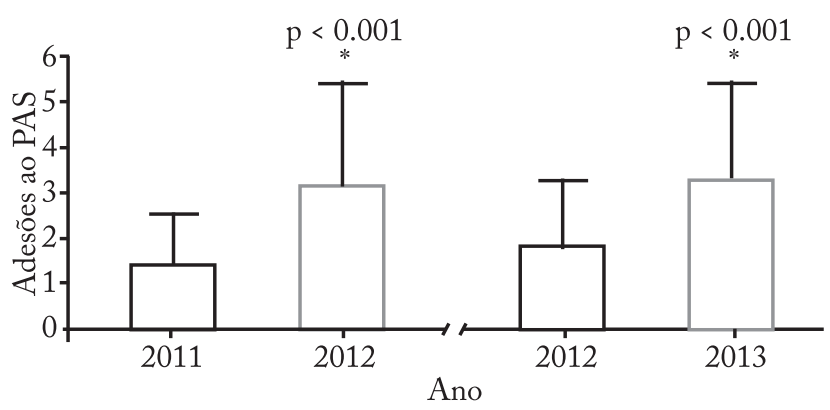

Figura 2 - Comparação entre o número médio de adesões ao Programa Academia da Saúde (PAS), em 2011 versus 2012 e em 2012 versus 2013.

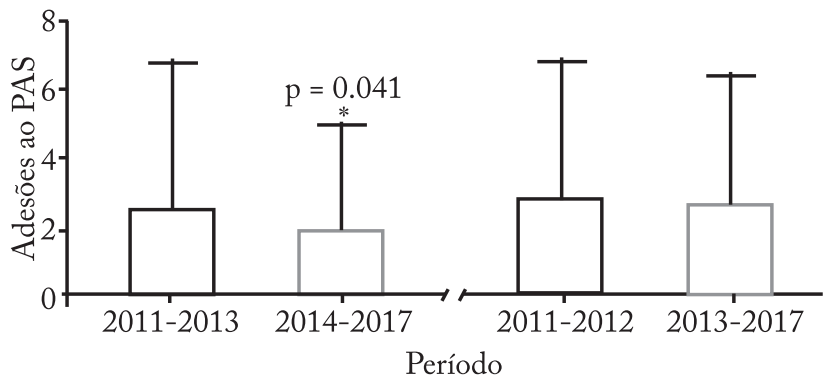

Figura 3 - Comparação por períodos entre o número médio de adesões ao Programa Academia da Saúde (PAS).

reu após a exclusividade de financiamento por EP. Além disso, embora todas as macrorregiões tivessem apresentado um percentual de finalização de obras acima de $60 \%$, o baixo percentual de custeio das academias da saúde pode indicar fragilidades na implantação do PAS.
Mesmo considerando as adesões canceladas, o cenário observado demonstra uma abrangência nacional expressiva em termos de municípios-PAS. O número de adesões e a capilaridade do PAS no Brasil podem beneficiar o campo da saúde pública se forem implementados satisfatoriamente. O PAS já foi considerado "o cartão de visita" da PS e da atividade física no SUS ${ }^{14}$, amenizando assim, o cenário de escassez de iniciativas preventivas e de PS.

A presença do componente político em programas que buscam oportunizar o acesso às atividades físicas é inegáve $1^{15}$, ainda mais quando dependem de recursos financeiros advindos da decisão legislativa. Nos dois primeiros anos de financiamento de EP, os resultados mostraram o direcionamento de novas adesões para municípios que já participavam. Isso também foi constatado no período de 2011 a 2013, especialmente para os municípios do Sudeste. Isso pode indicar que no primeiro momento de instauração das AEP, a concentração de adesões nos mesmos municípios foi facilitada, mas não se pode afirmar que a alteração do financiamento foi responsável por essa dinâmica, uma vez que na vigência de financiamento exclusivo de EP não há diferença estatística ao período anterior.

O financiamento das obras é peculiar na compreensão do PAS. Como já mencionado, o financiamento por EP advém da interpretação do que seriam gastos 
em saúde ${ }^{11}$. Por ser uma ação intersetorial, o PAS não poderia ser objeto de repasses pelo MS, conferindo ao legislativo o poder político na escolha dos municípios e do quantitativo de adesões, sem a observância de critérios técnicos, como situação epidemiológica e socioeconômica local. Em consonância com a teoria do PAS, municípios com maiores necessidades de prevenção de DCNT deveriam ter sido prioritários.

As macrorregiões não apresentaram diferenças percentuais marcantes na variável municípios-PAS. Sudeste foi a única com percentual abaixo de $50 \%$, mas como foi a macrorregião que de 2011 a 2013 mais recebeu adesões anuais adicionais nos mesmos municípios, permaneceu com o maior percentual de adesões. Essa região teve a maior proporção de cancelamentos, o que pode estar associado ao alto percentual de adesões em relação às demais macrorregiões. Esses cancelamentos vinculam-se à falta de interesse ou capacidade da gestão municipal em iniciar ou dar continuidade às obras. De acordo com a legislação em vigor, o procedimento de cancelamento é frequente para os casos em que prazos ou regras de monitoramento das obras não forem respeitados pela gestão municipal ${ }^{16}$. Portanto, mesmo que as adesões aumentem, o cenário real de implementação poderá sofrer alterações contínuas.

Dificuldade de gestão na condução das obras, com o consequente descumprimento de prazos, podem acentuar o desinteresse pela manutenção da adesão. Além disso, problemas licitatórios, mudanças de gestão municipal e insuficiência dos recursos financeiros podem prejudicar a implantação do PAS. Problemas associados à execução de obras são comuns na administração pública, todavia, mesmo havendo estratégias disponíveis para apoiá-los, são dificilmente solucionados por motivos técnicos, operacionais e orçamentários ${ }^{17}$. Como justificativa para essas dificuldades tem-se a descentralização das políticas do SUS, que de um lado aproximam a ação governamental da sociedade, mas, por outro, sofrem a limitação da capacidade do nível central em controlar ou apoiar a implementação de políticas, em observância ao princípio da autonomia municipal ${ }^{18}$.

Em consonância com as metas estabelecidas pelo governo, a adesão por obras impulsionou a expansão do PAS, com predomínio das AMS, apesar de terem ocorrido apenas em 2011 e 2012. Nordeste e Sudeste concentraram as maiores proporções, provavelmente em função do número superior de municípios. Além disso, a região Nordeste apresentou o menor percentual de obras concluídas, por outro lado, positivamente, a me- nor proporção de cancelamentos e o maior percentual de academias custeadas. Acreditamos que o interesse pelo PAS possa ter sido maior no Nordeste porque diversos municípios tiveram experiências pregressas de atividade física no SUS, sendo uma das macrorregiões mais beneficiadas pela extinta Rede Nacional de Atividade Física ${ }^{19}$. Pesquisas indicam que o Nordeste tem apresentado indicadores desfavoráveis para o controle e a prevenção das $\mathrm{DCNT}^{20,21}$. Contudo, essa região apresentou alto percentual de adesão, acrescido das características de funcionamento do PAS mais favoráveis do que nas outras macrorregiões ${ }^{22}$. Em médio e longo prazo, estudos avaliativos poderão apresentar resultados no enfrentamento das DCNT.

Em âmbito nacional, a expressiva expansão do PAS de 2011 a 2013, em termos de número de adesões e municípios-PAS, é contrastada com o baixo avanço nos anos de 2014 a 2017. A ruptura da expansão coincide com o período em que o financiamento dependeu exclusivamente de EP. Como as normas orçamentárias permitem a interferência do legislativo sobre a programação setorial ${ }^{23}$, o PAS pode ser visto como uma iniciativa governamental vulnerável a interesses políticos e restrições orçamentárias. Além disso, teve-se o processo de destituição da Presidente da República em meados de 2016, o que pode ter influenciado as políticas de saúde, no entanto, observamos que a queda da expansão do PAS já havia iniciado nos anteriores.

No Painel do Orçamento Federal ${ }^{24}$, percebe-se o aumento de recursos orçamentários de investimentos (EP e setorial) para o MS nos anos estudados, mas os recursos destinados ao PAS não seguiram essa tendência, ou seja, de 2011 a 2013 foram 8,5 bilhões e de 2014 a 2017 foram reduzidos para 4,6 bilhões. Considerando os números de atrasos e cancelamentos de obras, parece compreensível que o programa não tenha se beneficiado com esse aumento orçamentário, haja vista que investimentos em infraestrutura podem comprometer os ganhos políticos no curto prazo ${ }^{18}$.

$\mathrm{Na}$ região Norte, verificamos maior proporção de municípios-PAS, o que pode ser um resultado positivo considerando o número reduzido de oferta de atividade física na atenção primária ${ }^{21,25}$. Ainda sobre a região Norte, características geográficas e do sistema de saúde estadual podem ter influenciado o menor número de municípios-PAS no Amazonas em relação aos outros estados $^{26}$. Além disso, o Norte também apresentou o maior número de AEP. Historicamente essa macrorregião é sobrerrepresentada na Câmara dos Deputados, 
o que pode favorecê-la na captação de recursos ${ }^{27,28}$. Estudo em que foi analisada a representação dos estados no Congresso e os efeitos na alocação dos recursos federais (1997 a 2010), mostrou que o Norte recebeu o maior montante per capita de $\mathrm{EP}^{29}$.

A despeito das adversidades e complexidades na condução das obras do PAS, bem como os atrasos e as repactuações de prazos, a maioria das adesões estavam concluídas. Em estudo realizado em 2015, o número de obras sem conclusão era crítico $^{22}$. Os resultados aqui apresentados, portanto, indicam que houve avanço nesse aspecto, podendo ter contribuído para isso a existência de manual de infraestrutura ${ }^{30}$ e apoio técnico do MS.

O baixo percentual de academias da saúde custeadas indica que obras concluídas estavam inativas ou funcionavam sem o custeio. O repasse do custeio é um indicador administrativo para academia em funcionamento. $\mathrm{O}$ desconhecimento desse recurso pode afetar a sustentabilidade do PAS por demandar aportes financeiros da gestão municipal. Aproximadamente $90 \%$ dos municípios cofinanciam a implementação do PAS ${ }^{22}$, todavia os motivos dessa coparticipação ainda são desconhecidos. O baixo percentual de custeio também pode ter relação com o desinteresse municipal dado o valor de repasse (3 mil reais mensais) ser considerado insuficiente frente às exigências para a manutenção do recebimento (carga horária de funcionamento, contratação de profissionais específicos, entre outras) ou até mesmo pela falta de autonomia da gestão local ${ }^{31}$.

Algumas limitações devem ser consideradas na interpretação dos resultados. Apesar de serem resultados de diferentes momentos, o presente estudo possui delineamento transversal, o que implica no possível viés da causalidade reversa e limita as inferências entre possíveis causas e efeitos. Os resultados devem ser complementados com pesquisas que aprofundem a complexidade entre política, legislação pertinente e adesão pelos entes federados implícita no pacto interfederativo, considerando a implementação em contextos reais de descentralização. Além disso, seriam oportunos estudos sobre interferência das decisões legislativas e do processo orçamentário na definição de investimento para o PAS e outros programas de PS, esporte e lazer.

Com base nos resultados, percebemos uma capilaridade relevante do PAS no território nacional, todavia a adesão per si não representa a possibilidade de maior oferta de serviços de PS e prevenção de DCNT. A trajetória do processo de adesão até o funcionamento das academias demanda a observância e o cumprimento de diver- sas regras administrativas, aspectos que podem ser politicamente desinteressantes. Adesões foram canceladas e municípios deixaram de participar da implementação, bem como, um número expressivo de obras concluídas se tornaram infraestrutura sem custeio, e, caso estejam em funcionamento, são invisíveis para o governo federal.

Por fim, concluímos que o PAS está bem distribuído pelo país, com diferentes situações de implantação. $\mathrm{O}$ período de maior expansão foi de 2011 a 2012, quando predominou o financiamento do MS para as obras. De 2013 a 2017, após a inclusão do financiamento exclusivo por EP, o quantitativo de adesões não superou o dos dois primeiros anos. Diante do progressivo aumento das DCNT na população brasileira, o PAS permanece como uma das principais iniciativas governamentais na atenção primária e sua continuidade e expansão não podem estar atreladas a interesses políticos, mas sim a indicadores socioeconômicos e de saúde, para garantir a equidade e universalidade no acesso.

\section{Conflito de interesse}

Os autores declaram não haver conflito de interesse.

\section{Contribuições dos autores}

Tusset D, trabalhou na concepção do desenho do estudo, análise e interpretação dos dados, elaboração de todas as versões do artigo e aprovação da versão final. Merchan-Hamann E, contribuiu na concepção do desenho do estudo, interpretação dos dados, revisão crítica e aprovação da versão final. Calmon PCDP, atuou na concepção do desenho do estudo, revisão crítica e aprovação da versão final. Santos L, trabalhou na interpretação dos dados, revisão crítica e aprovação da versão final. Olkoski M, também trabalhou na interpretação dos dados, revisão crítica e aprovação da versão final.

\section{Referências}

1. Barros MBA, Francisco PMSB, Zanchetta LM, César CLG. Trends in social and demographic inequalities in the prevalence of chronic diseases in Brazil. PNAD: 2003-2008. Ciênc Saúde Colet. 2011;16(9):3755-68.

2. Malta DC, Morais Neto OL, Silva Junior JB. Apresentação do plano de ações estratégicas para o enfrentamento das doenças crônicas não transmissíveis no Brasil, 2011-2022. Epidemiol Serv Saúde. 2011;20(4):425-38.

3. World Health Organization. Nairobi Call to Action. 2009. Disponível em: https:/www.who.int/healthpromotion/ conferences/7gchp/documents/en/.

4. Kickbusch I. The Contribution of the World Health Organization to a New Public Health and Health Promotion. Am J Public Health. 2003;93(3):383-8.

5. Rocha DG, Alexandre VP, Marcelo VC, Rezende R, Nogueira JD, Sá RF. Processo de revisão da Política Nacional de Promoção da Saúde: múltiplos movimentos simultâneos. Ciênc Saúde Colet. 2014;19(11):4313-22. 
6. Neto JLF, Kind L, Resende MCC, Colen NS. Processos da construção da Política Nacional de Promoção da Saúde. Cad Saúde Pública. 2013;29(10):1997-2007.

7. Brasil. Política Nacional de Promoção da Saúde. Revisão da Portaria MS/GM no 687, de 30 de março de 2006. Disponível em: http://bvsms.saude.gov.br/bvs/publicacoes/ pnps_revisao_portaria_687.pdf

8. Carvalho FFB, Nogueira JAD. Práticas corporais e atividades físicas na perspectiva da Promoção da Saúde na Atenção Básica. Ciênc Saúde Colet. 2016;21(6):1829-38.

9. Brasil. Plano de ações estratégicas para o enfrentamento das doenças crônicas não transmissíveis (DCNT) no Brasil: 2011-2022. 1 edição. Brasília, DF: Ministério da Saúde; 2011. 154 p.

10. Brasil. Portaria no 2861, de 7 de novembro de 2013. Redefine o Programa Academia da Saúde no âmbito do Sistema Único de Saúde. 2013. Disponível em: https://bvsms.saude.gov.br/ bvs/saudelegis/gm/2013/prt2681_07_11_2013.html

11. Brasil. Regulamentação da Emenda Constitucional no 29, DE 2000 (Piso Constitucional de Aplicação em Saúde). Análise das principais inovações trazidas pela Lei Complementar no 141, de 13 de janeiro de 2012, que regulamentou a Emenda Constitucional no 29, de 2000. [Internet]. Câmara dos Deputados; 2012. Disponível em: http://www2.camara.leg. br/orcamento-da-uniao/estudos/2012/nt14.pdf

12. Tusset D, Santos L, Merchan-Hamann E, Calmon PCDP. Programa Academia da Saúde: correlação entre internações por doenças crônicas não transmissíveis e adesão nos municípios brasileiros, 2011-2017. Epidemiol Serv Saúde. 2020. Disponível em: http://scielo.iec.gov.br/pdf/ess/ v29n5/2237-9622-ess-29-05-e2019453.pdf

13. Brasil. Portaria no 1.707 , de 23 de setembro de 2016 . Redefine as regras e os critérios referentes aos incentivos financeiros de investimento para construção de polos; unifica o repasse do incentivo financeiro de custeio por meio do Piso Variável da Atenção Básica (PAB Variável); e redefine os critérios de similaridade entre Programas em desenvolvimento no Distrito Federal e nos Municípios e o Programa Academia da Saúde. [Internet]. 2016 p. 4. Disponível em: http://189.28.128.100/ dab/docs/portaldab/documentos/academia/Portaria $\% 20$ 1707\%20de\%2023\%2009\%202016\%20-\%20Redefine\%20 regras\%20Academia\%20da\%20Sade.pdf

14. Silva KL, Sena RR, Silva $P$, Magalhães RV, Tavares TS. Institucionalização de programas de promoção da saúde: definições na gestão municipal. Rev enferm UFPE on line. 2015;9(12):1190-7.

15. Maciel MG, Couto ACP. Programas Governamentais de Atividade Física: uma proposta de política pública. Rev PPP. 2018;11(22):55-79.

16. Brasil.Portaria GM n³81, de 6 de fevereiro de 2017 [Internet]. 2017 [citado 22 de outubro de 2018]. Disponível em: http:// www.lex.com.br/legis_27294556_PORTARIA_N_381_ DE_6_DE_FEVEREIRO_DE_2017.aspx

17. Ribeiro R. A lenta Evolução da Gestão de Obras Públicas no Brasil. E-Legis - Revista Eletrônica do Programa de Pós-Graduação da Câmara dos Deputados. 23 de junho de 2012;8(8):82-103.

18. Amorim Neto $O$, Simonassi AG. Bases políticas das transferências intergovernamentais no Brasil (1985-2004). Brazil J Polit Econ. 2013;33(4):704-25.
19. Amorim T, Knuth A, Cruz D, Malta D, Reis R, Hallal P. Descrição dos programas municipais de promoção da Atividade Física financiados pelo Ministério da Saúde. 2013;18(1):63-74.

20. Jaime PC, Stopa SR, Oliveira TP, Vieira ML, Szwarcwald CL, Malta DC. Prevalência e distribuição sociodemográfica de marcadores de alimentação saudável, Pesquisa Nacional de Saúde, Brasil 2013. Epidemiol Serv Saúde. 2015;24(2):267-76.

21. Ramos LR, Malta DC, Gomes GAO, Bracco MM, Florindo AA, Mielke GI, et al. Prevalence of health promotion programs in primary health care units in Brazil. Rev Saúde Públ. 2014;48(5):837-44.

22. Sá GBAR, Dornelles GC, Cruz KG, Amorim RCA, Andrade SSCA, Oliveira TP, et al. O Programa Academia da Saúde como estratégia de promoção da saúde e modos de vida saudáveis: cenário nacional de implementação. Ciênc Saúde Coletiva. 2016;21(6):1849-60.

23. Sanches OM. A atuação do Poder Legislativo no orçamento: problemas e imperativos de um novo modelo [Internet]. 1998 [citado 22 de março de 2019]. Disponível em: http://www2. senado.leg.br/bdsf/handle/id/360

24. Brasil. Painel do Orçamento Federal [Internet]. Sistema Integrado de Planejamento e Orçamento. 2019 [citado 22 de março de 2019]. Disponível em: https://www1. siop.planejamento.gov.br/QvAJAXZfc/opendoc. htm?document=IAS\%2FExecucao_Orcamentaria. qvw\&host=QVS\%40pqlk04\&anonymous=true

25. Gomes GAO, Kokubun E, Mieke GI, Ramos LR, Pratt M, Parra DC, et al. Characteristics of physical activity programs in the Brazilian primary health care system. Cad Saúde Pública. 2014;30(10):2155-68.

26. Garnelo L, Sousa ABL, Silva CO. Regionalização em Saúde no Amazonas: avanços e desafios. Ciênc Saúde Coletiva. 2017;22:1225-34.

27. Deda CC, Kauchakje S. Determinantes das transferências fiscais para as unidades municipais: efeitos da representação desproporcional dos distritos eleitorais brasileiros. Rev Adm Pública. 2019;53(1):150-72.

28. Nicolau JM. As Distorções na Representação dos Estados na Câmara dos Deputados Brasileira. Dados [Internet]. 1997 [citado 26 de março de 2019];40(3). Disponível em: http:// www.scielo.br/scielo.php?script $=$ sci_abstract\&pid $=$ S00 11-52581997000300006\&lng=en\&nrm=iso\&tlng=pt

29. Turgeon M, Cavalcante P. Desproporcionalidade da representação dos estados no Congresso Nacional e seus efeitos na alocação dos recursos federais. IPEA. 2014.

30. Brasil. Publicações do Programa Academia da Saúde [Internet]. Portal da Saúde. 2017 [citado 21 de outubro de 2019]. Disponível em: http://www.saude.gov.br/acoes-eprogramas/academia-da-saude/publicacoes

31. Mota PHS, Viana ALA, Bousquat A, Mota PH S, Viana ALA, Bousquat A. Relações federativas no Programa Academia da Saúde: estudo de dois municípios paulistas. Saúde Debate. 2016;40(108):64-73.

Recebido: $28 / 06 / 2020$

Aprovado: 20/11/2020 